\title{
Cyclic 5-membered disulfides are not selective substrates of thioredoxin reductase, but are opened nonspecifically by thiols
}

\author{
Jan G. Felber ${ }^{1}$, Lena Poczka ${ }^{1}$, Sander Busker ${ }^{2}$, Ulrike Theisen ${ }^{3}$, Lukas Zeisel ${ }^{1}$, Martin S. Maier ${ }^{1}$, Kristina Loy ${ }^{1}$, Christina Brandstädter ${ }^{4}$, Karoline \\ Scholzen ${ }^{2}$, Katja Becker ${ }^{4}$, Elias S. J. Arnér ${ }^{2,5}$, Julia Thorn-Seshold ${ }^{1}$, Oliver Thorn-Seshold ${ }^{1}$ \\ ${ }^{1}$ Department of Pharmacy, Ludwig-Maximilians University Munich, Butenandtstr. 5-13, 81377 Munich, DE. \\ ${ }^{2}$ Department of Medical Biochemistry, Karolinska Institut, Solnavägen 9, 17177 Stockholm, SE. \\ ${ }^{3}$ Zoological Institute, Cellular and Molecular Neurobiology, TU Braunschweig, Spielmannstr. 7, 38106 Braunschweig, DE. \\ ${ }^{4}$ Interdisciplinary Research Centre (IFZ), Justus-Liebig University Giessen, Heinrich-Buff-Ring 26-32, 35392 Giessen, DE. \\ ${ }^{5}$ Department of Selenoprotein Research, National Institute of Oncology, 1122 Budapest, HU.
}

\begin{abstract}
The cyclic five-membered disulfide 1,2-dithiolane has been used as the key element in numerous chemical biology probes. Contradictory views of this disulfide motif populate the literature: some reports describe it as being nonspecifically reduced, others as a highly specific substrate for thioredoxin reductase (TrxR). We show that 1,2-dithiolanes are nonspecifically reduced by a broad range of thiol reductants and redox-active proteins, and that their cellular performance is barely affected by TrxR inhibition or knockout. We conclude that inhibitor screenings and probe designs treating 1,2-dithiolanes as TrxR-selective substrates should be treated with caution and previous interpretations may need careful re-evaluation. Considering ring-opening polymerisation, and stringently interpreting assays involving the thiophilic gold-based inhibitor auranofin, are critical to assess 1,2-dithiolane's true behaviour. We present an approach to control against assay misinterpretation with reducible probes, to ensure that future TrxR-targeted designs are robustly evaluated for selectivity, and to better orient future research.
\end{abstract}

\section{INTRODUCTION}

Specific dithiol/disulfide-exchange reactions underlie a great number of crucial pathways in biology. Often, these are coordinated through conserved, highly specialised networks of oxidoreductases. $^{1}$ The thioredoxin reductase - thioredoxin (TrxR/Trx) system, and the glutathione reductase - glutathione glutaredoxin (GR/GSH/Grx) system, are central "nodes" in these networks. TrxR (nM cellular concentration) passes reducing equivalents from $\mathrm{NADPH}$ to the effector protein $\operatorname{Trx}(\mu \mathrm{M})$. Similarly, GR (nM) passes reducing equivalents from NADPH to the redox-active peptide GSH (mM), that can directly function as a cellular reductant or be further shuttled to the effector Grx proteins $(\mu \mathrm{M})$. These systems drive hundreds of redox reactions vital to cellular metabolism, and also regulate protein activity, proteinprotein interactions, and protein localisation by reversible dithiol/disulfide-type reactions. ${ }^{2}$ Their complex homeostasis is dysregulated in many diseases, particularly in autoimmune disorders and cancer, ${ }^{3}$ making Trx and TrxR promising therapeutic targets. ${ }^{4}$ Designing selective probes or substrates that report on or target these redox nodes, would enable abroad range of applications in both basic biological and applied biomedical research, and is therefore a subject of intense development both through genetic engineering and chemical biology approaches. ${ }^{5,6}$

Disulfides are the native substrates of these redox manifolds, and both linear and cyclic disulfides have been exploited as artificial substrates in biophysics, materials chemistry and chemical biology.

Driven by the high intracellular concentration of thiols (ca. $50 \mathrm{mM}$ total, ca. $5 \mathrm{mM}$ GSH) compared to low concentrations in plasma or in the extracellular space, linear disulfides undergo irreversible and nonspecific transthiolation and reduction in cells (Fig 1a). ${ }^{7}$ Linear disulfides are thus used for nonspecific intracellular release and/or activation of appended cargos, exploiting the cellular thiol pool.
By contrast, cyclic disulfides can exhibit quite different kinetics and thermodynamics for disulfide transthiolation or reduction, and may therefore display different specificity. Cyclic systems are found in nature, perhaps most remarkably in the epidithiodiketo-piperazine class of natural products (ETP)..$^{8-10}$ ETPs such as gliotoxin (Fig 1b) and chaetocin feature a near-planar diketopiperazine that is 1,4-bridged by a disulfide with an unfavourable CSSC dihedral angle of $0^{\circ}$ as compared to the more favourable dihedral angle found in linear disulfides $\left(90^{\circ}\right)$ or 6 membered cyclic disulfides $\left(60^{\circ}\right)$. ETPs were reported to inhibit a range of enzymes and cause a variety of toxic cellular effects, but these often poorly reproducible bioactivities are now understood as relatively nonspecific chemical reactivity of their highly strained disulfide. ${ }^{11}$

A particularly important cyclic disulfide is the 5-membered 1,2-dithiolane (in this work also termed SS-50). This motif underlies the critical cellular redox cofactor lipoic acid, as well as being found in several natural products (Fig 1c), ${ }^{12,13}$ and it has emerged as a motif of general interest within the last decade. ${ }^{14-16}$

The strained 1,2-dithiolane is kinetically labile to transthiolation, ${ }^{18}$ which is likely the reason for its biological role as a redox cofactor. The disulfide's opening/reduction kinetics have made it the focus of numerous chemical biology approaches, although these have been predicated on two mutually contradictory views of its cellular behaviour, which in this paper we will examine and resolve.

Following one view, 1,2-dithiolane has been cast as an easily, nonspecifically, and irreversibly transthiolated and/or reduced motif. Whitesides' systematic disulfide investigations highlighted that its unfavourable CSSC dihedral angle of ca. $30^{\circ}$ destabilises it by more than $8 \mathrm{~kJ} / \mathrm{mol}$ relative to linear disulfides $\left(90^{\circ}\right)$, and that its reduction potential (ca. -240 to $-270 \mathrm{mV}$ ) is not significantly below that of linear disulfides (ca. $-230 \mathrm{mV}$ ). In the 1950s, Fava observed that alkyl thiolates transthiolate 1,2-dithiolane ca. 5000 times faster 
than linear aliphatic disulfides. ${ }^{19}$ Creighton reported that its transthiolation by the vicinal dithiol dithiothreitol (DTT) is over 100 times faster still, ${ }^{20}$ and Whitesides showed that in DMSO, this rate is only 100 -fold slower than the diffusion limit. ${ }^{21}$ With favourable thermodynamics and high kinetic lability, 1,2-dithiolanes readily polymerise at low concentrations by nucleophile-catalysed ring-opening polymerisation, in particular in the presence of thiols. ${ }^{22,23}$ Matile showed that the sterically less shielded 1,2-dithiolane in asparagusic acid derivatives (two primary thiols) polymerise even more easily than do lipoic acid derivatives, ${ }^{24}$ giving them valuable applications exploiting proximity-inducedpolymerisation. $^{25}$

The intrinsic lability of 1,2-dithiolanes has also been extensively applied for thiol-mediated cellular uptake systems. Promoted by strain, 1,2-dithiolanes are transthiolated at the cell surface, then are internalised through a series of dynamic covalent reactions with membrane and intracellular thiols. Attaching the strained disulfide to a cargo can therefore dramatically enhance its cellular uptake rate (Fig 1d; overview in Fig S1a). ${ }^{17,24,26-29}$ It will be important to note that this process may be affected by thiophilic inhibitors: uptake rates could be decreased 5-fold by treating cells with thiol-alkylating or thiol-oxidising reagents, or instead enhanced by treatment with reducing agents. Other recent studies further demonstrate that 1,2-dithiolanes undergo fast strain-driven cross-linking or polymerisation initiated by thiols or other nucleophiles. ${ }^{30,31}$

In contrast however, 1,2-dithiolanes have also been reported as reduction-sensing units with a remarkable selectivity for TrxR. The fluorogenic TRFS probes $^{14,16,32,33}$ and prodrugs $^{15}$ have been commercialised and extensively reviewed ${ }^{34-38}$ (Fig 1e; overview in Fig S1b). These probes have since been used to study the role of TrxR in Parkinson's disease ${ }^{39}$ and stroke ${ }^{33}$, and have been employed for mechanistic validation of putative TrxR inhibitors during screening approaches. ${ }^{16}$

Which is the real situation? 1,2-dithiolane cannot simultaneously be TrxR-selective and yet highly and nonspecifically reactive. To develop a systematic understanding of redox biology, it is necessary to clarify such fundamental disagreements, and reveal why such contradictory results could arise.

Towards this goal, we have investigated the novel 1,2dithiolane-based reduction-sensing probe SS-50-PQ. We show that (a) its 5-membered cyclic disulfide is nonspecifically reduced by a broad range of monothiols, dithiols, proteins and enzymes, and (b) its cellular processing does not exclusively require TrxR.

Taken together, we conclude that 1,2-dithiolane-based probes are not selective for cellular TrxR, so the previous work interpreting the performance of TRFS probes as TrxR reporters ${ }^{14-16,32,33,39}$ will benefit from re-evaluation. We speculate that strained disulfides are best understood as probes to monitor thiol-mediated uptake rates, and can be legitimately applied for enhanced delivery and nonspecific activation of trigger-cargo-systems, which opens up promising avenues for chemical biology. We also outline a strategy to control against assay misinterpretations with reducible probes, to promote progress towards a robust and useful toolset of probes for redox biology. a

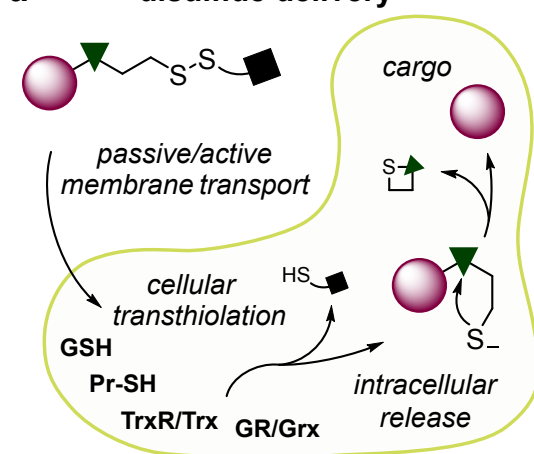

b

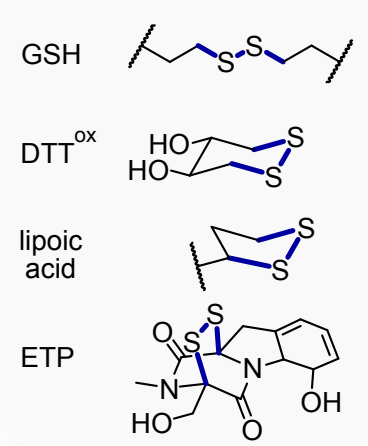

disulfide strain

IS- $\sim 90^{\circ}$

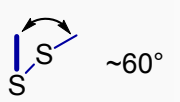

$\left.\right|_{S} ^{\prime} \quad-30^{\circ}$

$\sum_{s}^{r} \sim 0^{\circ}$
C<smiles>O=C(O)CCCC1CCSS1</smiles>

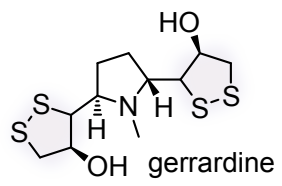

kottamide d strain-promoted cellular uptake

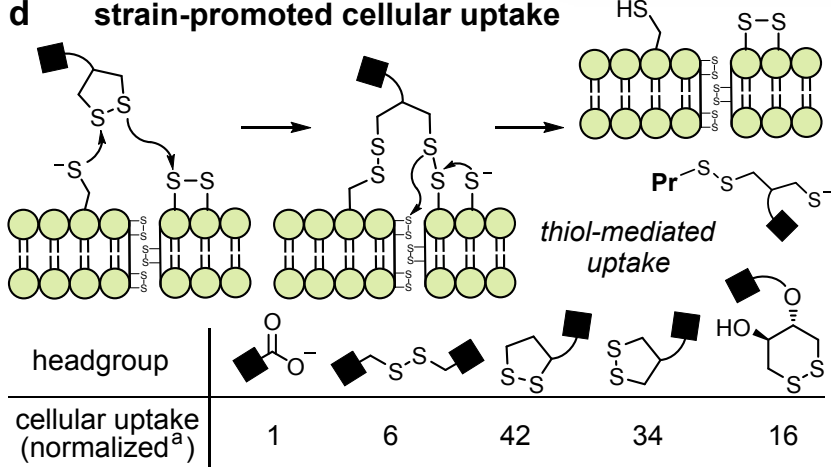

e<smiles>CCCCN1C(=O)c2ccc3c(cccc3c2NC(=O)OC2CSCS2)C1=O</smiles>

1,2-dithiolane-based probes<smiles></smiles>

S-Gem<smiles>[R]CCC(=O)OCCCNC(=O)Nc1ccc2c(C(F)(F)F)cc(=O)oc2c1</smiles>

TRFS-red

Fast-TRFS

Figure 1: 1,2-dithiolanes in chemical biology. (a) Principle of disulfide-based cellular delivery: irreversible cleavage of linear disulfides after cell entry leads to intracellular cargo release (Pr-SH: intracellular protein thiol). (b) Increasing ring strain from linear, to 6-membered and 5-membered disulfides, to ETPs. (c) Strained 1,2-dithiolanes in natural products. (d) Fast, irreversible and nonspecific transthiolation of 1,2dithiolanes with exofacial protein thiols permits rapid dynamic exchange cascades enhancing cellular cargo uptake. ${ }^{\left[{ }^{[a]}\right.}$ : data from Matile et al. ${ }^{17}$ (e) 1,2-dithiolane-based probes that have been reported as selective cellular substrates of thioredoxin reductase. 


\section{RESULTS AND DISCUSSION}

Probe design: We aimed to explore the properties of 1,2-dithiolanes using a reduction-activated probe. Reductionactivated probes and prodrugs are typically trigger-cargo designs, where a reduction-sensitive trigger is connected to the cargo while masking a key functional group. Trigger reduction then results in a fragmentation reaction that restores activity by unmasking that key functional group. This concept has been used for a range of imaging agent ${ }^{40,41}$ and drug ${ }^{42-44}$ cargos.

The TRFS probes are also designed as trigger-cargo constructs with 1,2-dithiolane as the redox sensor, attached to aniline fluorophores through a carbamate (TRFS-red, TRFS-green; Fig 1e) or urea (Fast-TRFS; Fig 1e). For the carbamate probes, disulfide reduction and thiol cyclisation releases the active aniline fluorophore; though the urea probe operates without aniline unmasking, and the fluorescence turn-on mechanism is not fully understood. Generally, most trigger-cargo disulfide probes employ aniline rather than phenol cargos (Fig S1b). While aniline carbamates have high hydrolytic stability, ${ }^{16}$ phenol-releasing designs would in many ways be more attractive targets, due to a large scope of cargos and to their improved release kinetics. Therefore, to test the reduction selectivity of 1,2-dithiolane with a rapidly-responding probe - and at the same time to establish a modular design that allows delivering a wide range of agents in the future - we created the novel phenol-releasing probe SS-50-PQ(Fig 2a).

The SS-50-PQ design has several advantages. As a tertiary carbamate, this probe cannot decompose by $\mathrm{E}_{1 \mathrm{cB}}$ elimination, ${ }^{45,46}$ avoiding the instability ${ }^{16}$ that has blocked previous phenol-releasing 1,2-dithiolane probes. The choice of 2-(2'-hydroxyphenyl)-4(3H)quinazolinone (PQ-OH) as the cargo, ensures a complete-off-to-on signal readout for carbamate cleavage, making it a sensitive and easily interpreted sensor of disulfide cleavage.

Following disulfide transthiolation and/or reduction and thiolate cyclisation, expulsion of the electron-poor phenolate (Fig 2a) results in PQ-OH precipitating upon reaching its low aqueous solubility limit (ca. $0.5 \mu \mathrm{M}) \cdot^{47,48}$ Only in the solid-state precipitate does PQ-OH exhibit its large-Stokes-shift ESIPT-based fluorescence, that involves intramolecular transfer of the phenolic hydrogen $(\mathrm{ex} / \mathrm{em} 360 / 530 \mathrm{~nm})$. Therefore, fluorescence is unambiguously due to cyclisation-mediated cargo release, and combined with the large Stokes shift, the system gives excellent signal-to-background ratios of typically $>100$ without needing background subtraction.

Probe synthesis: 1,2-dithiolane 6 was prepared using an approach initially reported by Raines ${ }^{49}$ followed by $\mathrm{N}$-methylation and Boc-deprotection (Fig 2b). All intermediates containing the 5-membered cyclic disulfide showed degradation upon standing, that we presume to be linear polydisulfide oligomers. This occured even without clear stimuli (e.g. stirring in dichloromethane). The cyclic monomer could typically be recovered by stirring in dilute methanolic solution, although isolating the monomer from this solution while avoiding re-polymerisation was not straightforward. The $N$-methylation step suffered particular tendency towards polymerisation, until we found that the monomeric product could be extracted from methanol by hexane washes (see Supporting Information). The final fluorogenic probe SS-50-PQ was assembled by carbamate coupling with PQ-OH.

1,2-dithiolane is unstable in probes: Polymerisation was also observed for probe stock solutions in DMSO. Their maximal fluorescence, determined by applying a standardised reducibility test (aq. buffer, $\mathrm{pH} 7.4,10 \mathrm{eq}$ of the quantitative disulfide reductant tris(carboxyethyl) phosphine, TCEP) decreased over time. We understood this as a consequence of polymerisation, since the hydrophobic, polymeric degradation products would have decreased accessibility to solvated reductants. Fresh probe stocks were therefore prepared immediately for each assay from powdered solid, then assayed for quality with comparison to calibration curves established by precipitating the theoretical amount of PQ-OH. Only stocks yielding TCEP-related signals within $10 \%$ of the calibration intensity were used in assays. In fact, we re-prepared SS-50-PQ five times during this research to maintain high-quality stocks.

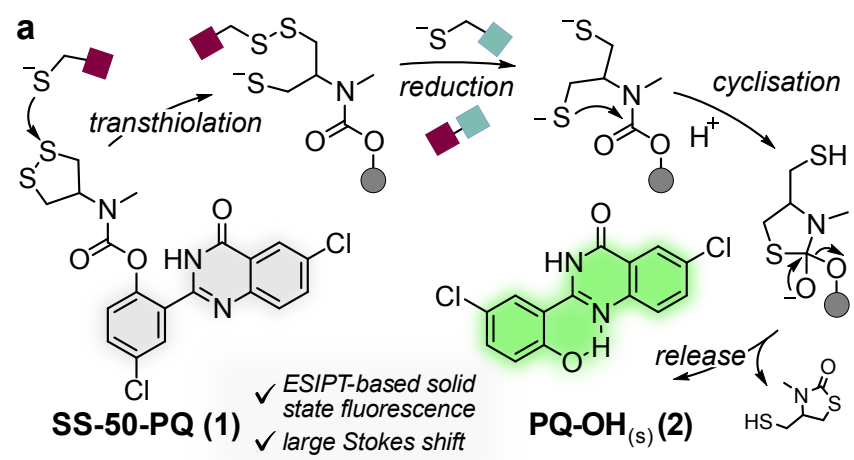

b
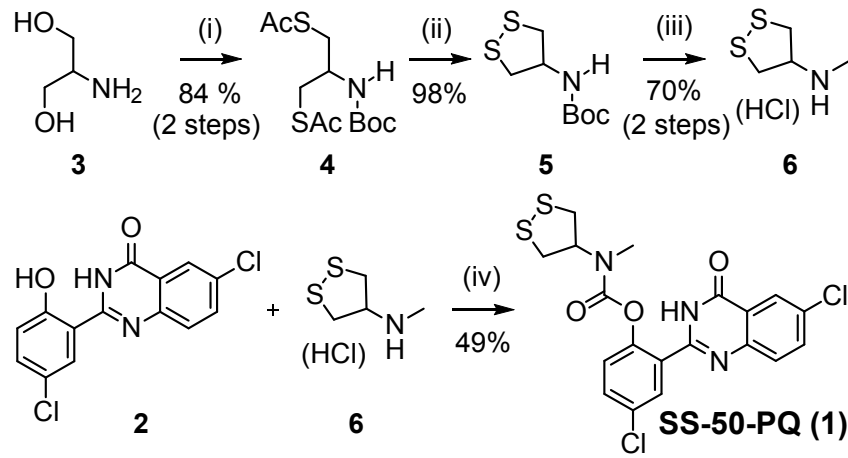

Figure 2: 1,2-dithiolane probe design and synthesis. (a) After reduction of the 1,2-dithiolane in SS-50-PQ thiolate cyclisation releases the precipitating phenol PQ-OH that gives ESIPT-based fluorescence in the solid state. (b) Synthesis of SS-50-PQ: (i) $\mathrm{Boc}_{2} \mathrm{O}, \mathrm{NEt}_{3}$, dioxane $/ \mathrm{H}_{2} \mathrm{O}$, r.t., $15 \mathrm{~h}$ (96\%) then either $\mathrm{MsCl}$, py, DCM followed by $\mathrm{KSAc}$, acetone, $60^{\circ} \mathrm{C}, 2 \mathrm{~h}(89 \%)$, or $\mathrm{HSAc}, \mathrm{PPh}_{3}, \mathrm{DIAD}, \mathrm{THF}, 0^{\circ} \mathrm{C}$ to r.t., $15 \mathrm{~h}(88 \%)$. (ii) $\mathrm{KOH}$, $\mathrm{MeOH}$, open to air, r.t., $15 \mathrm{~h}$ (98\%). (iii) $\mathrm{MeI}, \mathrm{NaH}, \mathrm{DMF}, 0^{\circ} \mathrm{C}$ to r.t., $0.5 \mathrm{~h}$ (70\%). (iv) PQ-OH, triphosgene, $\mathrm{NEt}_{3}, \mathrm{DCM}, 0^{\circ} \mathrm{C}$ to r.t., $1 \mathrm{~h}$; then 6, $\mathrm{NEt}_{3}, \mathrm{DCM}, 0^{\circ} \mathrm{C}$ to r.t., $1 \mathrm{~h}(49 \%)$.

1,2-dithiolane is nonspecifically reduced by various thiols: A trigger can only be enzyme-selective in the cellular context, if it resists transthiolation or reduction by the cellular monothiol background (ca. $50 \mathrm{mM}$, of which ca. $5 \mathrm{mM} \mathrm{GSH}{ }^{50-52}$ ) Hence, we began testing the potential for selectivity by performing cell-free incubations of SS-50-PQ $(10 \mu \mathrm{M})$ with GSH. Probe "sensitivity or resistance" to challenge by a species, is often cited according to a measurement at a single challenge concentration at a single timepoint. However, this allows inconsistent or selectively chosen results to oversimplify or misrepresent a more complex situation. To provide a useful characterisation of probe resistance to monothiols, we titrated GSH 
over a wide concentration range $(0.01$ to $10 \mathrm{mM})$ and collected time-course fluorescence data (Fig 3a). For more meaningful representations, we normalised the signals at each timepoint to the maximum fluorescence time-course value at that timepoint (TCEP), and also calculated dose-response curves at various endpoints (Fig 3b; see Fig S2). This normalisation is important and can be generally recommended: it helps to separate kinetics of fragmentation from kinetics of reduction (otherwise, slow fragmentation can hide reductive sensitivity); and it allows direct comparison of experiments relative to their theoretical maxima. We observed strong, fast probe response to even subphysiological GSH levels. The GSH concentrations causing half-maximal fluorescence ("EC ${ }_{50}{ }^{\mathrm{GSH}}$ ") were $\lesssim 1 \mathrm{mM}$ (Fig 3b; Fig S2). This indicates that 1,2-dithiolane probes can be rapidly and fully reduced by cellular GSH concentrations, even without enzyme catalysis involved.

We also screened other monothiol reductants, e.g. cysteine (Cys), N-acetylcysteine (NAC), N,N-dimethyl-cysteamine (MEDA), and cysteamine (CA) and found fast probe activation (Fig 3c-d) with similar concentrations and kinetics compared to GSH. This suggests that 1,2-dithiolane is generally instable to monothiols, so that probes derived from it might be rapidly activated by the intracellular thiol background. Matching expectations from Creighton $^{20}$ and Whitesides ${ }^{21}$, the probe was quantitatively and rapidly triggered by equimolar vicinal dithiol DTT. We controlled for release by mechanisms other than transthiolation/reductiontriggered cyclisation, using serine (Ser) and glutathione disulfide (GSSG). The probe was entirely stable to non-reductive degradation as e.g. aminolysis, highlighting the stability of the tertiary phenolic carbamate, and supporting that transthiolation/reduction is its pathway for signal generation (Fig 3c).

In summary, these assays show that 1,2-dithiolanes do not resist uncatalysed reduction by monothiols, even at subphysiological concentrations. This provides initial evidence that probes using 5-membered cyclic disulfides may not be enzyme-selective in the biological context.

1,2-dithiolane is nonspecifically reduced by redox-active proteins and enzymes: We next tested probe reduction with redox proteins from the Trx/TrxR and Grx/GSH/GR systems. Each protein has multiple isoforms, as has been excellently reviewed ${ }^{53} \mathrm{We}$ employed recombinant human Trx1 and Trx2; the thioredoxinrelated protein TRP14, which features a vicinal dithiol/disulfide redox-active site that is also reduced by TrxR; the oxidoreductases TrxR1, TrxR2 and GR; and human vicinal dithiol glutaredoxins Grx1 and Grx2. Both Trxs and Grxs have orders of magnitude higher cellular concentrations (ca. $10 \mu \mathrm{M}$ ) than their upstream TrxR and GR partners (ca. $20 \mathrm{nM}$ ), so we reflected these concentrations in our assays. SS-50-PQ was challenged with combinations of proteins from these redox systems. To study whether probes were reduced by the effectors Trx or Grx, and/or by direct reaction with the upstream reductants TrxR or GR, we compared assays using both effectors and upstream reductants, against assays employing only upstream reductants or only effectors (TrxR/GR assays included NADPH; $\mathrm{GR}+\mathrm{Grx}$ assays included $10 \mu \mathrm{M}$ GSH for Grx reduction; see Supporting Information).

The 1,2-dithiolane probe was nonspecifically reduced, with Trx1, Trx2, TRP14, Grx1, Grx2, TrxR1 and TrxR2 all rapidly reaching high conversion. Only the highly GSSG-specific enzyme GR gave no signal (Fig 3e, Fig S3). Time-courses even showed that both enzymatic cascades (TrxR/Trx and GR/GSH/Grx; Fig 3f) have identical activation profiles.

Taken together, the 1,2-dithiolane probe is nonselectively and nonenzymatically triggered by GSH and monothiols at subphysiological concentrations, as well as by a broad range of dithiol/disulfide-type proteins and enzymes. The systematic variation and titration of chemo- and bioreductants, and the examination of both time-course and endpoint data, show that 1,2dithiolane is not a TrxR-selective substrate in a cell-free setting. a

GSH resistance
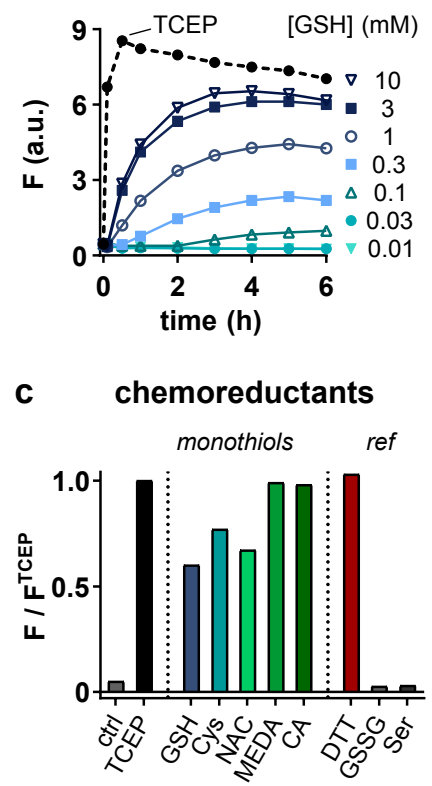

e

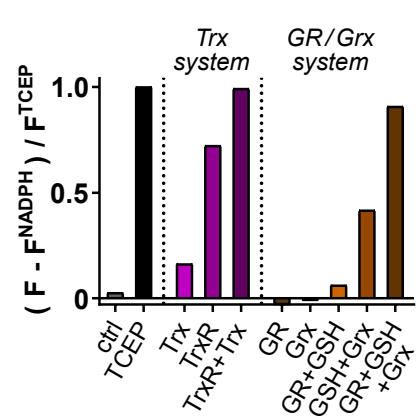

b dose response

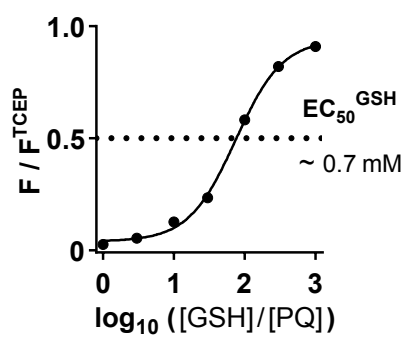

d kinetics

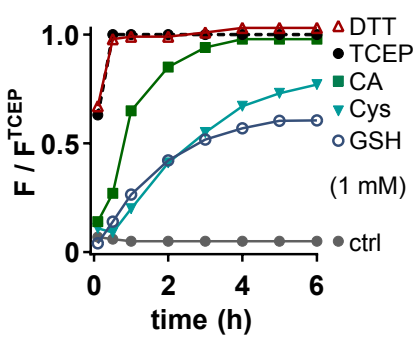

f

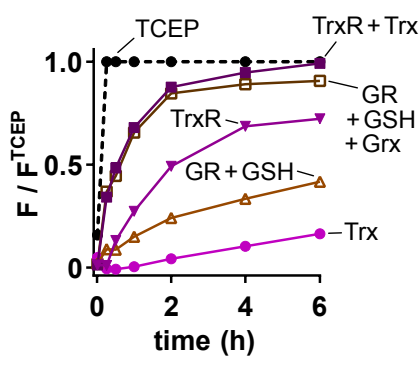

Figure 3: 1,2-dithiolane probe SS-50-PQ is activated by a range of chemical and biological reductants. (a) Fluorescence time-courses of SS-50-PQ (10 $\mu \mathrm{M}$ in TE-buffer) exposed to GSH. (b) Dose-response plot for GSH triggering of SS-50-PQ (10 $\mu \mathrm{M}$ in TE-buffer, $6 \mathrm{~h}$ at $37^{\circ} \mathrm{C}$ ). (c-d) Chemical reductant assays. Normalised signal from SS-50-PQ $(10 \mu \mathrm{M}$ in TE-buffer) when challenged with monothiol reductants $(1 \mathrm{mM})$ or reference reagents ( $1 \mathrm{mM}$ DTT, GSSG and Ser, or $100 \mu \mathrm{M}$ TCEP). (c) Single timepoint results $\left(6 \mathrm{~h}\right.$ at $\left.37^{\circ} \mathrm{C}\right)$ and (d) selected kinetics. (e-f) Redox enzyme assays. Normalised signal from SS-50-PQ (10 $\mu \mathrm{M}$ in TE-buffer) challenged with TrxR/Trx or GR/GSH/Grx network proteins $(20 \mathrm{nM}$ TrxR1/GR, $10 \mu \mathrm{M}$ Trxl/Grxl, $100 \mu \mathrm{M}$ GSH as indicated; $100 \mu \mathrm{M}$ NADPH in all TrxR/GR assays). (e) Single timepoint results $\left(6 \mathrm{~h}\right.$ at $\left.37^{\circ} \mathrm{C}\right)$ and $(\mathbf{f})$ selected kinetics. 
The 1,2-dithiolane probe is cellularly activated: We tested the cellular activation of SS-50-PQ in four cell lines: HeLa cervical cancer, A549 lung cancer, Jurkat T-cell lymphoma cells, and mouse embryonic fibroblasts (MEF). All cell lines rapidly generated welldefined fluorescent precipitates of PQ-OH. Fluorescence platereader quantification showed a nearly linear increase of signal in the first $6 \mathrm{~h}$ (Fig 4a) and the signal was concentration-dependent (Fig 4b), indicating that no saturation effects are operative. The solid preciptates of PQ-OH were intracellularly localised and visible in most cells (Fig 4c). Because platereader data and microscopy images can misrepresent population-level response, we used flow cytometry to collect single-cell-resolved statistics of probe activation. Though this is unusual for small molecule probes, it is possible with SS-50-PQbecause the solid PQ-OH precipitate is cellularly retained during cell fixation. These data showed a monomodal fluorescence intensity distribution with $>60 \%$ of cells exhibiting strong PQ-OH fluorescence (Fig 4d, Fig S5). a

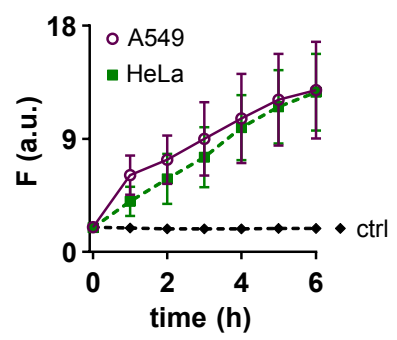

\section{b}

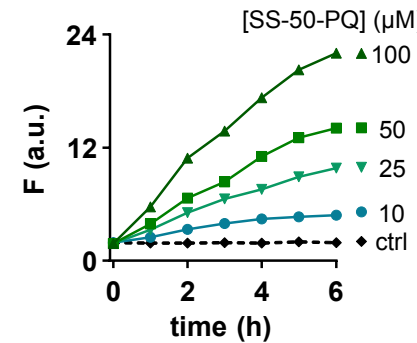

single-cell statistics
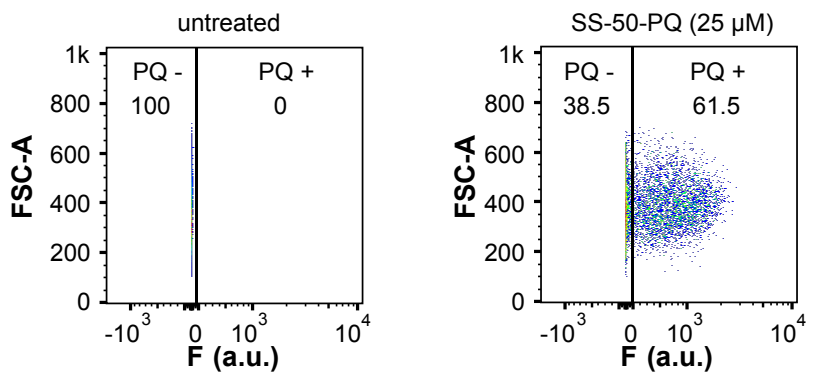

The 1,2-dithiolane probe is activated in vivo: At this point, we considered it valuable to apply SS-50-PQ in a simple in vivo model, to test three design goals of our phenolic carbamate probe system: (a) zero signal background due to mechanistic quenching in the probe and high Stokes shift of the released fluorophore; (b) no spontaneous cargo release; due to the hydrolytic robustness of the tertiary carbamate; and (c) cellular retention of $\mathbf{P Q - O H}$ precipitates for high-spatial-resolution imaging in vivo. Therefore, we incubated zebrafish zygotes and embryos up to 3 days post fertilisation (dpf) in media containing SS-50-PQ and acquired time-courses by epifluorescence and live confocal microscopy (Fig 4e, Fig S7-S8). Probe activation began within two hours, with intriguing localisation of the marked cells, although the interpretation of this pattern would need additional investigation and we do not believe that it is connected to TrxR activity. Most pleasingly however, all three probedesign goals were achieved: high-contrast images were obtained without background manipulation, with precise resolution that will be particularly important for future investigations.

C

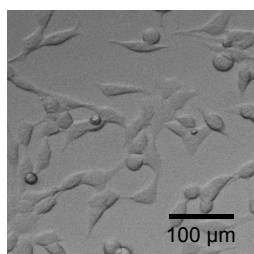

ctrl

e

\section{fluorescence microscopy}

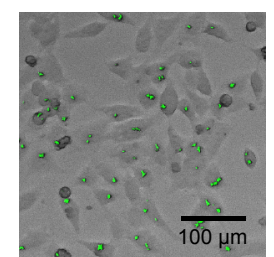

$25 \mu \mathrm{M}$

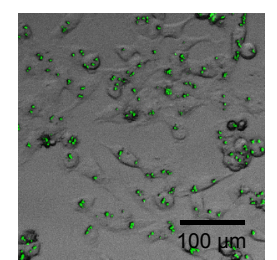

$50 \mu \mathrm{M}$ in vivo imaging

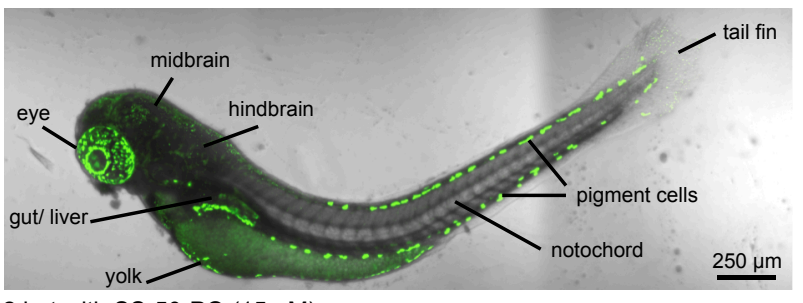

$2 \mathrm{hpt}$ with SS-50-PQ $(15 \mu \mathrm{M})$

Figure 4: The 1,2-dithiolane-based probe SS-50-PQ is activated in cellulo and in vivo. (a) Cellular fluorescence time-course with SS-50-PQ ( $50 \mu \mathrm{M}$; HeLa and A549 cells; 3 independent experiments). (b) Dose-dependency of cellular fluorescence time-courses with SS-50PQ (10-100 $\mu \mathrm{M}$; HeLa cells). (c) Representative microscopy images of SS-50-PQ-treated HeLa cells show fluorescent intracellular solid precipitates of PQ-OH. (d) Flow cytometry-based single-cell statistics of cellular fluorescence after SS-50-PQ treatment (25 $\mu$ M; Jurkat Tcells). (e) Fluorescence image of a zebrafish embryo (3 dpf), after $2 \mathrm{~h}$ treatment with SS-50-PQ $(15 \mu \mathrm{M})$.

Cellular probe activation is not blocked by TrxR suppression: We then tested the TrxR-specificity of cellular probe activation in several ways. Cells cultured without selenium supplementation may not fully incorporate SeCys in TrxR, lowering cellular TrxR activity. ${ }^{54}$ However, we did not observe significant dependency of SS-50-PQ signal upon $\mathrm{Na}_{2} \mathrm{SeO}_{3}$ supplementation (Fig 5a). We more stringently evaluated TrxR-dependency using a TrxR1 knockout MEF cell line ( $\left.\operatorname{TrxR}^{-/}\right)$compared to its control $\left(\operatorname{TrxR} 1^{\mathbb{A} / \mathbb{1}}\right)$. Their signal time-courses were indistinguishable, i.e. probe activation is independent of TrxR1 expression (Fig $\mathbf{4 b}$ ). We then explored whether chemical inhibition of TrxR affects signal generation, using the selective TrxR inhibitors TRi- 1 and TRi-3. ${ }^{55}$ Cells pretreated with TRis gave similar probe signals as the noinhibitor controls (with partial inhibition observed for high- concentration experiments above $2 \mu \mathrm{M}$ ) and depending on the time of pre-incuation, regardless of whether this was evaluated as in timecourse or endpoint (Fig 5c-d; and Fig S4) population-average assays, or with single-cell statistics (Fig 5e; and Fig S5-6). The inhibition effect was evaluated for different duration of the cells' pre-treatment to exlucde, that the cells recover from TrxR inhibition and their biological consequences within a time window of $15 \mathrm{~h}$. Therefore, neither TrxR inhibition nor TrxR knockout greatly alter the cellular activation of SS-50-PQ. Together with the cell-free results showing rapid and nonspecific activation of 1,2dithiolane by cellular thiols, we conclude that cellular activation of 1,2-dithiolane probes does not exlusively report on TrxR activity. 
Why might 1,2-dithiolanes have been previously proposed as TrxR-selective substrates? Our cell-free and cellular assays had however shown that 1,2-dithiolane is not selective for TrxR. However, previous studies of 1,2-dithiolane probes have claimed excellent TrxR selectivity. This claim was based on assays where exposure of cells or lysates to auranofin (AF; Fig 5) dosedependently reduced fluorescence signals as compared to untreated controls. ${ }^{39}$ We were intrigued to study AF more closely, to understand if/why it may have led to misinterpretation of selectivity, and to assess what information monomeric SS-50-type probes really provide in the biological context.

$\mathrm{AF}$ is a thiophilic $\mathrm{Au}(\mathrm{I})$ complex that is popularly used as an inhibitor of TrxR. AF binds TrxR in cell-free assays and in cells. However, AF is more generally a "potent thiol-reactive species" 56 that is also reported to bind at least 20 other thiol protein targets, and its therapeutic mechanisms of action are widely accepted to be unresolved, ${ }^{57}$ although it is clear that TrxR is one of its major targets. ${ }^{57}$ Notably, AF is a particularly strong binder of membrane thiols, possibly driven by its lipophilicity. ${ }^{58}$

We confirmed that cells pretreated with $\mathrm{AF}(0.1$ to $4 \mu \mathrm{M})$ had decreased probe processing, seen by both single-cell (Fig 5e, Fig S5-6) and population average (Fig 5d,f) measurements. However, this does not necessarily indicate that probe processing occurs through TrxR. Given AF's polypharmacology, the dithiolane's cell-free nonselectivity, and the minor effects of nonAu-based TrxR modulation on probe activation (Fig 5a-e), we were motivated to look more critically into this result. Conclusively, even in a fully cell- and enzyme-free setting, AF catalytically suppressed the fluorescence generated upon TCEP treatment, without a clear dose-dependence (Fig 5g). Therefore, signal suppression in the cellular assay cannot be used to argue that TrxR, or any other cellular target of auranofin, might be a selective reductant of 1,2-dithiolane. a

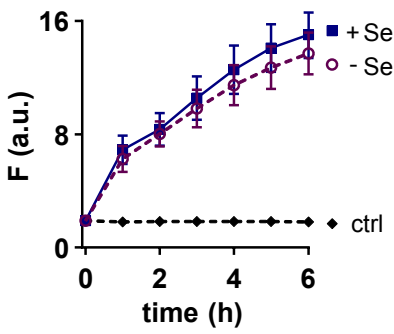

d

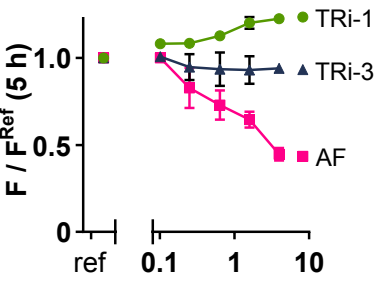

$\log _{10}([$ inhibitor $])(\mu \mathrm{M})$ b TrxR knockout

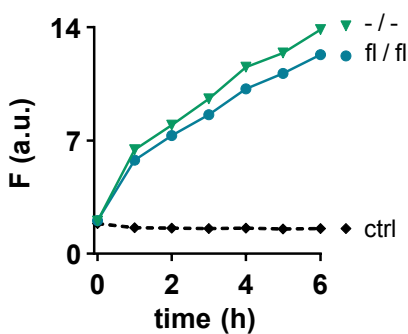

e single-cell statistics

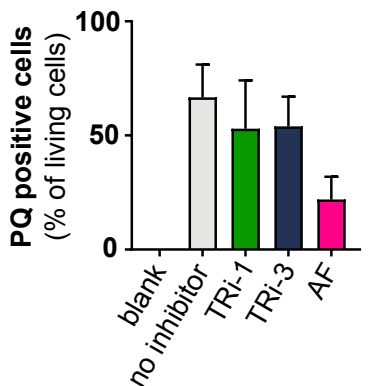

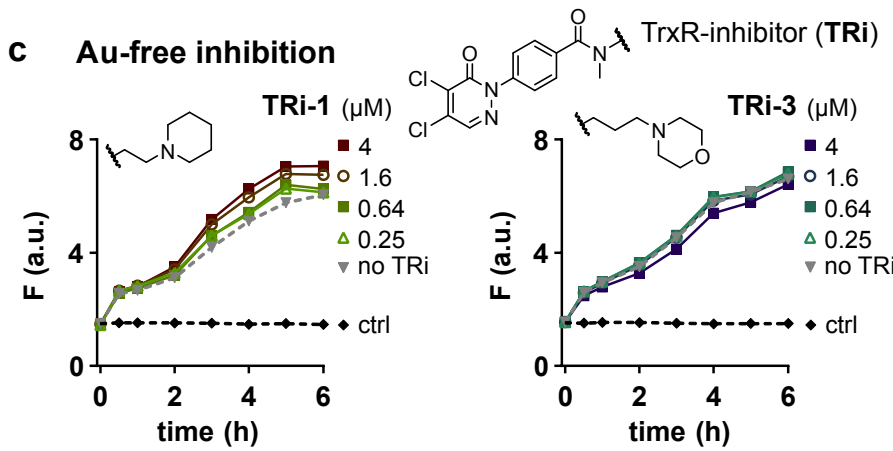

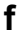

g cell-free inhibition

Au-based inhibition (HeLa cells)

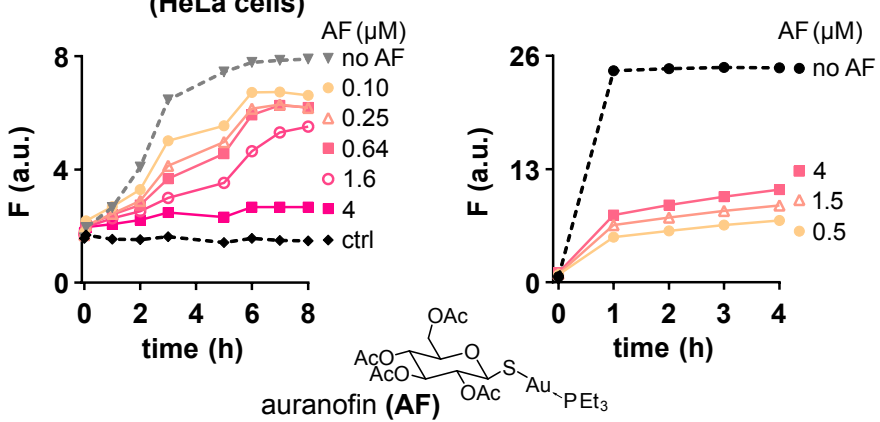

Figure 5: The activation of 1,2-dithiolane probes in cells does not require TrxR. (a) Cellular fluorescence time-courses in A549 cells cultured with/without $\mathrm{Na}_{2} \mathrm{SeO}_{3}$-supplemented medium ( $50 \mu \mathrm{M}$ SS-50-PQ). (b) Cellular fluorescence time-courses of TrxR1-knockout (-/-) and -wildtype (fl/fl) MEF cells. (c) Cellular fluorescence time-courses of HeLa cells pretreated with the gold-free TrxR inhibitors TRi-1 and TRi-3 (25 $\mu$ M SS-50-PQ 15 h pre-treatment). (d) Cellular fluorescence dose-reponse plots for cells pre-treated with TRi-1, TRi-3 and AF ( $25 \mu \mathrm{M}$ SS-50-PQ $15 \mathrm{~h}$ pre-treatment). (e) Flow cytometry-based single-cell statistics of cellular fluorescence $(25 \mu \mathrm{M}$ SS-50-PQ in HeLa cells optionally pretreated $(15 \mathrm{~h})$ with TRi-1, TRi-3 or the Au(I)-based inhibitor auranofin $(\mathrm{AF} ; 4 \mu \mathrm{M})$, compared to reference fluorescence ("blank", no SS-50-PQ) and to uninhibited cells ("no inhibitor"). (f) Fluorescence time-courses of HeLa cells pretreated with AF (25 $\mu$ M SS-50-PQ). (g) Cell-free, enzyme-free fluorescence time-course of TCEP $(100 \mu \mathrm{M})$ reduction of SS-50-PQ $(25 \mu \mathrm{M}$ in TE-buffer $)$ after pretreatment of the probe stock with AF (at r.t. for $2 \mathrm{~h}$ ).

We present two hypotheses that plausibly explain why AF suppresses signal from SS-50-PQ that are consistent with 1,2-dithiolane being a nonspecific thiol probe, for which TrxR is not a significant cellular reductant. (1) The strained disulfide probe usually benefits from dynamic covalent exchange enhancement of cellular delivery, therefore AF's binding to membrane thiols ${ }^{58}$ suppresses cellular delivery and signal. Similar suppression of delivery of strained disulfide substrates has been achieved by thiophilic organic alkylators of exofacial thiols. ${ }^{17}$ This delivery inhibition is consistent with literature findings and our data showing that reaching the intracellular environment, 1,2dithiolanes are nonspecificially opened by thiols (Fig 3 ), and it could be responsible for some or most of the cellular signal suppression by auranofin. (2) AF reacts with 1,2-dithiolane probes 
to suppress signal by both directly and catalytically diminishing their reducibility. Literature reports indeed show that $\mathrm{Au}(\mathrm{I})$-(thiolate)(phosphine) complexes react with disulfides, initially forming triethylphosphine oxide and the thioglucose-goldthiolate complex. ${ }^{59,60}$ Since $\mathrm{Au}(\mathrm{I})$-bis(thiolate) complexes undergo facile net ligand exchange with disulfides, they mediate thiol-disulfide exchange reactions ${ }^{61}$, which in the case of 1,2-dithiolane would trigger ring-opening polymerisation. ${ }^{62}$ Polymerisation of the 1,2-dithiolane-based probe would generate hydrophobic polymeric products, which (as literature suggests ${ }^{63}$ ) are not reducible by even such strong aqueous reductants as TCEP, so suppressing fluorescent signal generation. This would explain auranofin's cell-free (Fig 5g) signal suppression, and can even be sufficient to explain its cellular signal suppression, that we and others have observed.

Motivated by the known broad spectrum of auranofin's targets and effects, it would be beneficial to at least take design precautions (e.g. cell-free controls testing AF-probe interactions, or using TRi inhibitors for competition assays) to identify likely confounding factors before advancing targets of strained disulfide probes based on AF experiments. We think it would be best to avoid Au-based reagents altogether, due to the high chance of any assay result being misinterpreted based on a populous literature of AF targets with no clear consensus. These may be valuable guides for analysis of cellular redox probe selectivities in the future.

\section{CONCLUSIONS}

Specific dithiol/disulfide-exchange reactions are central to biology, and engineered disulfides exploiting these reaction manifolds are finding applications from chemical biology probes to biophysics and materials chemistry. Linear disulfides have been known for decades as nonspecifically transthiolated/reduced substrates, and often used for intracellular release of cargos. 1,2-dithiolanes have emerged as substrates of interest in chemical biology ${ }^{14-16}$, although it has remained contentious whether its reduction is enzyme-selective, or nonspecific. We answered this question by studying the biochemical and biological performance of the novel 1,2-dithiolane-based redox probe SS-50-PQ. Here, the disulfide was integrated in a stable, modular design that can be adapted to release arbitrary phenols: in this case, a releaseactivated fluorescent cargo, that we could even use in flow cytometry studies and for cell-resolved imaging in live embryos.

A rigorous methodology of reductant titrations, enzyme panel screenings, and inhibitor/knockout cell experiments conclusively demonstrated the nonspecific reduction-based cleavage of the 1,2-dithiolane. While 1,2-dithiolane can indeed be rapidly opened by $\operatorname{TrxR}^{18}$ (Fig 3c), it is also rapidly opened by many other reducing thiol species. Therefore, in cells, it is highly unlikely to selectively report on TrxR, instead of on more abundantly present reductants (Fig 3). The cellular data show that neither TrxR knockout, nor TrxR inhibition by recently developed Au-free inhibitors, greatly modulate the cellular signal obtained from the 1,2-dithiolane probe (Fig 5). We pool literature references with our data to show that inhibition of cellular activation of probes based on 5-membered cyclic disulfides by treatment with auranofin can result from at least two plausible, precedented processes that do not involve TrxR: i.e., delivery inhibition by binding exofacial thiols, and suppressing probe reducibility by catalysing dithiolane ring-opening polymerisation. Taken together, we conclude that 1,2-dithiolane is an easily and nonspecifically transthiolated and/or reduced motif, that is not a TrxR-specific substrate.

On the other hand, there may be immediate rewards if 1,2-dithiolane-based reduction probes are properly re-evaluated. This would help maintain a clear literature, avoiding nonspecific electrophilic pan-assay interference compounds (PAINS) being falsely identified as TrxR-substrate hits, and could perhaps allow 1,2-dithiolane probes or prodrugs instead to find valuable applications as simple, modular systems for thiol-mediated cellular uptake and activation, impacting research in cell penetration and assisted uptake (extended discussion as a Supplementary Note in the Supporting Information).

Diversifying trigger structures to reach redox substrates that are selective for the key oxidoreductases remains a central goal for research in the field. Our modular probe system already ensured zero signal background, excellent hydrolytic robustness, and cellular marking for high-spatial-resolution imaging; these beneficial features might be transitioned to powerful redox probes, if more specific reduction-sensing units can be identified and installed as trigger motifs. In particular, selective substrates for GR, TrxR or Trx would, at last, allow researchers to unveil the dynamics that drive these major dithiol/disulfide-type enzyme systems within cells. By identifying and avoiding problematic and nonselective substrate types, chemical development may instead deliver selective and robust redox chemotypes for bioreductive probe and prodrug research; these will be reported in due course.

\section{ASSOCIATED CONTENT}

\section{Supporting Information}

Synthesis, analysis, biological evaluations, extended discussion (PDF)

\section{AUTHOR INFORMATION}

\section{Corresponding Author}

- $\quad$ oliver.thorn-seshold@cup.lmu.de

ORCIDs

Jan G. Felber: 0000-0002-5010-9624

Elias Arnér: 0000-0002-4807-6114

Julia Thorn-Seshold: 0000-0002-4879-4159

Oliver Thorn-Seshold: 0000-0003-3981-651X

\section{Author Contributions}

J.G.F. performed synthesis, chemical and photochemical analysis, chemoreductant and enzymatic cell-free studies, cellular studies, and coordinated data assembly. L.P. performed cellular inhibitor studies and FACS-based single-cell statistics. S.B., K.S. and C.B. performed enzymatic specificity screenings. U.T. performed zebrafish embyro studies. K.L. performed cellular studies. L.Z. and M.M. performed synthesis and analysis. K.B. and E.A. supervised enzymatic specificity screenings. J.T.-S. performed cellular studies, supervised cell biology and coordinated data assembly. O.T.-S. designed the concept and experiments, supervised all other experiments, coordinated data assembly and wrote the manuscript.

\section{ACKNOWLEDGMENT}

This research was supported by funds from the German Research Foundation (DFG: SFB 1032 project B09 number 
201269156, SFB TRR 152 project P24 number 239283807, SPP 1926 project number 426018126, and Emmy Noether grant 400324123 to O.T.-S.; SPP 1710 project BE $1540 / 23-2$ to K.B. (now transferred to Stefan Rahlfs)); LMUExcellent (Junior Researcher Fund to O.T.-S.); the Munich Centre for NanoScience initiative (CeNS, to O.T.-S.); and from Karolinska Institutet, The Knut and Alice Wallenberg Foundations, The Swedish Cancer Society, The Swedish Research Council and the Hungarian Thematic Excellence Programme (TKP2020-NKA-26) to E.S.J.A..J.G.F. thanks the Studienstiftung des deutschen Volkes for support through a PhD scholarship; J.T.-S. thanks the Joachim Herz Foundation for fellowship support. We thank Peter Mayer (LMU) for X-ray crystallography; Marcus Conrad (Helmholtz Centre, Munich) for MEF and knockout MEF cell lines; Hartmann Harz and Christoph Jung (LMU microscopy platforms) for assistance with microscopy facilities; and Matt Fuchter (ICL), Kate Carroll (Scripps), and the attendees of the SPP 1710 conference Thiol-Based Redox Switches in 2019 for their supportive and collegial discussions.

\section{REFERENCES}

(1) Arnér, E. S. J.; Holmgren, A. Physiological Functions of Thioredoxin and Thioredoxin Reductase: Thioredoxin and Thioredoxin Reductase. Eur J Biochem 2000, 267 (20), 6102-6109. https://doi.org/10.1046/j.14321327.2000.01701.x.

(2) Jones, D. P.; Sies, H. The Redox Code. ARS 2015, 23 (9), 734-746. https://doi.org/10.1089/ars.2015.6247.

(3) Arnér, E. S. J.; Holmgren, A. The Thioredoxin System in Cancer. Seminars In Cancer Biology 2006, 16 (6), 420-426. https://doi.org/10.1016/j.semcancer.2006.10.009.

(4) Arnér, E. S. J. Perspectives of TrxR1-Based Cancer Therapies. In Oxidative Stress; Elsevier, 2020; pp 639-667. https://doi.org/10.1016/B978-0-12-818606-0.00031-6.

(5) Hanson, G. T.; Aggeler, R.; Oglesbee, D.; Cannon, M.; Capaldi, R. A.; Tsien, R. Y.; Remington, S. J. Investigating Mitochondrial Redox Potential with Redox-Sensitive Green Fluorescent Protein Indicators. J Biol Chem 2004, 279 (13), 13044-13053. https://doi.org/10.1074/jbc.M312846200.

(6) Schwarzländer, M.; Dick, T. P.; Meyer, A. J.; Morgan, B. Dissecting Redox Biology Using Fluorescent Protein Sensors. ARS 2016, 24 (13), 680-712.

https://doi.org/10.1089/ars.2015.6266.

(7) Lee, M. H.; Yang, Z.; Lim, C. W.; Lee, Y. H.; Dongbang, S.; Kang, C.; Kim, J. S. Disulfide-Cleavage-Triggered Chemosensors and Their Biological Applications. Chemical Reviews 2013, 113 (7), 5071-5109. https://doi.org/10.1021/cr300358b.

(8) Welch, T. R.; Williams, R. M. Epidithiodioxopiperazines. Occurrence, Synthesis and Biogenesis. Nat Prod Rep 2014, $31 \quad$ (10), 1376-1404. https://doi.org/10.1039/C3NP70097F.

(9) Takahashi, M.; Takemoto, Y.; Shimazu, T.; Kawasaki, H.; Tachibana, M.; Shinkai, Y.; Takagi, M.; Shin-ya, K.; Igarashi, Y.; Ito, A.; Yoshida, M. Inhibition of Histone H3K9 Methyltransferases by Gliotoxin and Related Epipolythiodioxopiperazines. Journal Of Antibiotics 2012, 65 (5), 263-265. https://doi.org/10.1038/ja.2012.6.

(10) Zong, L.; Bartolami, E.; Abegg, D.; Adibekian, A.; Sakai, N.; Matile, S. Epidithiodiketopiperazines: Strain-Promoted
Thiol-Mediated Cellular Uptake at the Highest Tension. ACS-CS 2017, 3 (5), 449-453.

https://doi.org/10.1021/acscentsci.7b00080.

(11) Cherblanc, F. L.; Chapman, K. L.; Brown, R.; Fuchter, M. J. Chaetocin Is a Nonspecific Inhibitor of Histone Lysine Methyltransferases. NChB 2013, 9 (3), 136-137. https://doi.org/10.1038/nchembio.1187.

(12) Appleton, D. R.; Copp, B. R. Kottamide E, the First Example of a Natural Product Bearing the Amino Acid 4-Amino-1,2Dithiolane-4-Carboxylic Acid (Adt). Tet Lett 2003, 44 (50), 8963-8965. https://doi.org/10.1016/j.tetlet.2003.10.008.

(13) Kato, A.; Okada, M.; Hashimoto, Y. Occurrence of Gerrardine in Cassipourea Guianensis. JNP 1984, 47 (4), 706-707. https://doi.org/10.1021/np50034a024.

(14) Zhang, L.; Duan, D.; Liu, Y.; Ge, C.; Cui, X.; Sun, J.; Fang, J. Highly Selective Off-On Fluorescent Probe for Imaging Thioredoxin Reductase in Living Cells. JACS 2014, 136 (1), 226-233. https://doi.org/10.1021/ja408792k.

(15) Li, X.; Hou, Y.; Meng, X.; Ge, C.; Ma, H.; Li, J.; Fang, J. Selective Activation of a Prodrug by Thioredoxin Reductase Providing a Strategy to Target Cancer Cells. Angewandte Chemie International Edition 2018, 57 (21), 6141-6145. https://doi.org/10.1002/anie.201801058.

(16) Li, X.; Zhang, B.; Yan, C.; Li, J.; Wang, S.; Wei, X.; Jiang, X.; Zhou, P.; Fang, J. A Fast and Specific Fluorescent Probe for Thioredoxin Reductase That Works via Disulphide Bond Cleavage. NComm 2019, 10 (1), 2745. https://doi.org/10.1038/s41467-019-10807-8.

(17) Gasparini, G.; Sargsyan, G.; Bang, E.-K.; Sakai, N.; Matile, S. Ring Tension Applied to Thiol-Mediated Cellular Uptake. Angewandte Chemie International Edition 2015, 54 (25), 7328-7331. https://doi.org/10.1002/anie.201502358.

(18) Lothrop, A. P.; Ruggles, E. L.; Hondal, R. J. No Selenium Required: Reactions Catalyzed by Mammalian Thioredoxin Reductase That Are Independent of a Selenocysteine Residue. Biochem 2009, 48 (26), 6213-6223. https://doi.org/10.1021/bi802146w.

(19) Fava, A.; Iliceto, A.; Camera, E. Kinetics of the ThiolDisulfide Exchange. JACS 1957, 79 (4), 833-838. https://doi.org/10.1021/ja01561a014.

(20) Creighton, T. E. Interactions between Cysteine Residues as Probes of Protein Conformation: The Bisulphide Bond between Cys-14 and Cys-38 of the Pancreatic Trypsin Inhibitor. Journal Of Molecular Biology 1975, 96 (4), 767 776. https://doi.org/10.1016/00222836(75) $90151-5$

(21) Singh, R.; Whitesides, G. M. Degenerate Intermolecular Thiolate-Disulfide Interchange Involving Cyclic FiveMembered Disulfides Is Faster by .Apprx.103 than That Involving Six- or Seven-Membered Disulfides. JACS 1990, $112 \quad$ (17), 6304-6309. https://doi.org/10.1021/ja00173a018.

(22) Singh, R.; Whitesides, G. M. Thiol-Disulfide Interchange. In Sulphur-Containing Functional Groups; 1993; pp 633-658 (Wiley).

(23) Burns, J. A.; Whitesides, G. M. Predicting the Stability of Cyclic Disulfides by Molecular Modeling: Effective Concentrations in Thiol-Disulfide Interchange and the Design of Strongly Reducing Dithiols. JACS 1990, 112 (17), 6296-6303. https://doi.org/10.1021/ja00173a017. 
(24) Laurent, Q.; Sakai, N.; Matile, S. The Opening of 1,2Dithiolanes and 1,2-Diselenolanes: Regioselectivity, Rearrangements, and Consequences for Poly(Disulfide)s, Cellular Uptake and Pyruvate Dehydrogenase Complexes. Helv Chim 2019, 102 (2), e1800209. https://doi.org/10.1002/hlca.201800209.

(25) Sakai, N.; Lista, M.; Kel, O.; Sakurai, S.; Emery, D.; Mareda, J.; Vauthey, E.; Matile, S. Self-Organizing Surface-Initiated Polymerization: Facile Access to Complex Functional Systems. JACS 2011, 133 (39), 15224-15227. https://doi.org/10.1021/ja203792n.

(26) Abegg, D.; Gasparini, G.; Hoch, D. G.; Shuster, A.; Bartolami, E.; Matile, S.; Adibekian, A. Strained Cyclic Disulfides Enable Cellular Uptake by Reacting with the Transferrin Receptor. JACS 2017, 139 (1), 231-238. https://doi.org/10.1021/jacs.6b09643.

(27) Chuard, N.; Gasparini, G.; Moreau, D.; Lörcher, S.; Palivan, C.; Meier, W.; Sakai, N.; Matile, S. Strain-Promoted ThiolMediated Cellular Uptake of Giant Substrates: Liposomes and Polymersomes. Angewandte Chemie International Edition 2017, 56 (11), 2947-2950.

https://doi.org/10.1002/anie.201611772.

(28) Carmine, A.; Domoto, Y.; Sakai, N.; Matile, S. Comparison of Lipoic and Asparagusic Acid for Surface-Initiated Disulfide-Exchange Polymerization. Chemistry - A European Journal 2013, 19 (35), 11558-11563. https://doi.org/10.1002/chem.201301567.

(29) Chuard, N.; Poblador-Bahamonde, A. I.; Zong, L.; Bartolami, E.; Hildebrandt, J.; Weigand, W.; Sakai, N.; Matile, S. Diselenolane-Mediated Cellular Uptake. Chem Sci 2018, 9 (7), 1860-1866.

https://doi.org/10.1039/C7SC05151D.

(30) Barcan, G. A.; Zhang, X.; Waymouth, R. M. Structurally Dynamic Hydrogels Derived from 1,2-Dithiolanes. JACS 2015, 137 (17), 5650-5653. https://doi.org/10.1021/jacs.5b02161.

(31) Scheutz, G. M.; Rowell, J. L.; Ellison, S. T.; Garrison, J. B.; Angelini, T. E.; Sumerlin, B. S. Harnessing Strained Disulfides for Photocurable Adaptable Hydrogels. Macromolecules 2020, 53 (10), 4038-4046. https://doi.org/10.1021/acs.macromol.0c00604.

(32) Ma, H.; Zhang, J.; Zhang, Z.; Liu, Y.; Fang, J. A Fast Response and Red Emission Probe for Mammalian Thioredoxin Reductase. Chemical Communications 2016, 52 (81), 12060 12063. https://doi.org/10.1039/C6CC04984B.

(33) Zhao, J.; Qu, Y.; Gao, H.; Zhong, M.; Li, X.; Zhang, F.; Chen, Y.; Gan, L.; Hu, G.; Zhang, H.; Zhang, S.; Fang, J. Loss of Thioredoxin Reductase Function in a Mouse Stroke Model Disclosed by a Two-Photon Fluorescent Probe. Chemical Communications 2020, 56 (90), 14075-14078. https://doi.org/10.1039/D0CC05900E.

(34) Zhang, J.; Li, X; Han, X.; Liu, R.; Fang, J. Targeting the Thioredoxin System for Cancer Therapy. Trends In Pharmacological Sciences 2017, 38 (9), 794-808. https://doi.org/10.1016/j.tips.2017.06.001.

(35) Zhang, B.; Liu, Y.; Li, X.; Xu, J.; Fang, J. Small Molecules to Target the Selenoprotein Thioredoxin Reductase. Chemistry - An Asian Journal 2018, 13 (23), 3593-3600. https://doi.org/10.1002/asia.201801136.
(36) Zhang, J.; Zhang, B.; Li, X.; Han, X.; Liu, R,; Fang, J. Small Molecule Inhibitors of Mammalian Thioredoxin Reductase as Potential Anticancer Agents: An Update. Medicinal Research Reviews 2019, 39 (1), 5-39. https://doi.org/10.1002/med.21507.

(37) Zhang, J.; Duan, D.; Osama, A.; Fang, J. Natural Molecules Targeting Thioredoxin System and Their Therapeutic Potentials. ARS 2020. https://doi.org/10.1089/ars.2020.8213.

(38) Zhang, B.; Zhang, J.; Peng, S.; Liu, R.; Li, X.; Hou, Y.; Han, $\mathrm{X}$; Fang, J. Thioredoxin Reductase Inhibitors: A Patent Review. Expert Opinion On Therapeutic Patents 2017, 27 (5), 547-556. https://doi.org/10.1080/13543776.2017.1272576.

(39) Liu, Y.; Ma, H.; Zhang, L.; Cui, Y.; Liu, X.; Fang, J. A Small Molecule Probe Reveals Declined Mitochondrial Thioredoxin Reductase Activity in a Parkinson's Disease Model. Chemical Communications 2016, 52 (11), 22962299. https://doi.org/10.1039/C5CC09998F.

(40) Hong, K.-H.; Kim, D. I.; Kwon, H.; Kim, H.-J. A Fluoresceinylcarbonate-Based Fluorescent Probe for the Sensitive Detection of Biothiols in a HEPES Buffer and Its Cellular Expression. RSC Adv 2014, 4 (2), 978-982. https://doi.org/10.1039/C3RA42935K.

(41) Lim, C. S.; Masanta, G.; Kim, H. J.; Han, J. H.; Kim, H. M.; Cho, B. R. Ratiometric Detection of Mitochondrial Thiols with a Two-Photon Fluorescent Probe. JACS 2011, 133 (29), 11132-11135. https://doi.org/10.1021/ja205081s.

(42) Vrudhula, V. M.; MacMaster, J. F.; Li, Z.; Kerr, D. E.; Senter, P. D. Reductively Activated Disulfide Prodrugs of Paclitaxel. Bioorg Med Chem Lett 2002, 4.

(43) Henne, W. A.; Doorneweerd, D. D.; Hilgenbrink, A. R.; Kularatne, S. A.; Low, P. S. Synthesis and Activity of a Folate Peptide Camptothecin Prodrug. BMCL 2006, 16 (20), 5350-5355. https://doi.org/10.1016/j.bmcl.2006.07.076.

(44) Lee, S. H.; Kohn, H. Cyclic Disulfide C(8) Iminoporfiromycin: Nucleophilic Activation of a Porfiromycin. JACS 2004, 126 (13), 4281-4292. https://doi.org/10.1021/ja030577r.

(45) Ghosh, A. K.; Brindisi, M. Organic Carbamates in Drug Design and Medicinal Chemistry. JMC 2015, 58 (7), 28952940. https://doi.org/10.1021/jm501371s.

(46) Vacondio, F.; Silva, C.; Mor, M.; Testa, B. Qualitative Structure-Metabolism Relationships in the Hydrolysis of Carbamates. Drug Metabolism Reviews 2010, 42 (4), 551589. https://doi.org/10.3109/03602531003745960.

(47) Machida, T.; Dutt, S.; Winssinger, N. Allosterically Regulated Phosphatase Activity from Peptide-PNA Conjugates Folded Through Hybridization. Angewandte Chemie International Edition 2016, 55 (30), 8595-8598. https://doi.org/10.1002/anie.201602751.

(48) Rando, G.; Winssinger, N.; Lindberg, E.; Anzola, M. New Compounds and Uses Thereof for Detection of Target Molecules in a Sample. US 2019/0315713 A1.

(49) Lukesh, J. C.; Palte, M. J.; Raines, R. T. A Potent, Versatile Disulfide-Reducing Agent from Aspartic Acid. JACS 2012, 134 (9), 4057-4059. https://doi.org/10.1021/ja211931f. 
(50) Wu, G.; Fang, Y.-Z.; Yang, S.; Lupton, J. R.; Turner, N. D. Glutathione Metabolism and Its Implications for Health. Journal Of Nutrition 2004, 134 (3), 489-492. https://doi.org/10.1093/jn/134.3.489.

(51) Griffith, O. W. Biologic and Pharmacologic Regulation of Mammalian Glutathione Synthesis. Free Radical Biology Medicine 1999, 27 (9-10), 922-935. https://doi.org/10.1016/S0891-5849(99)00176-8.

(52) Jones, D. P. Redox Potential of GSH/GSSG Couple: Assay and Biological Significance. In Methods in Enzymology, 2002; pp 93-112 (Academic Press).

https://doi.org/10.1016/S0076-6879(02)48630-2.

(53) Fridovich, I. Antioxidant Enzymes. In Redox Biochemistry; 2007; pp 49-134 (Wiley).

https://doi.org/10.1002/9780470177334.ch3.

(54) Peng, X.; Xu, J.; Arnér, E. S. J. Thiophosphate and Selenite Conversely Modulate Cell Death Induced by Glutathione Depletion or Cisplatin: Effects Related to Activity and Sec Contents of Thioredoxin Reductase. Biochemical Journal 2012, 447 (1), 167-174. https://doi.org/10.1042/BJ20120683.

(55) Stafford, W. C.; Peng, X.; Olofsson, M. H.; Zhang, X.; Luci, D. K.; Lu, L.; Cheng, Q.; Trésaugues, L.; Dexheimer, T. S.; Coussens, N. P.; Augsten, M.; Ahlzén, H.-S. M.; Orwar, O.; Östman, A.; Stone-Elander, S.; Maloney, D. J.; Jadhav, A.; Simeonov, A.; Linder, S.; Arnér, E. S. J. Irreversible Inhibition of Cytosolic Thioredoxin Reductase 1 as a Mechanistic Basis for Anticancer Therapy. SciTransMed 2018, 10 (428).

(56) Cassini, A.; Messori, L. Molecular Mechanisms and Proposed Targets for Selected Anticancer Gold Compounds. Current Topics In Medicinal Chemistry 201 1, 11 (21), 2647-2660. https://doi.org/10.2174/156802611798040732.

(57) Saei, A. A.; Gullberg, H.; Sabatier, P.; Beusch, C. M.; Johansson, K.; Lundgren, B.; Arvidsson, P. I.; Arnér, E. S. J.; Zubarev, R. A. Comprehensive Chemical Proteomics for Target Deconvolution of the Redox Active Drug Auranofin. RedBiol 2020, 101491. https://doi.org/10.1016/j.redox.2020.101491.

(58) Snyder, R. M.; Mirabelli, C. K.; Crooke, S. T. Cellular Interactions of Auranofin and a Related Gold Complex with Raw 264.7 Macrophages. Biochemical Pharmacology 1987, 36 (5), 647-654. https://doi.org/10.1016/00062952(87)90715-5.

(59) Albert, A.; Brauckmann, C.; Blaske, F.; Sperling, M.; Engelhard, C.; Karst, U. Speciation Analysis of the Antirheumatic Agent Auranofin and Its Thiol Adducts by LC/ESI-MS and LC/ICP-MS. Journal Of Analytical Atomic Spectrometry 2012, 27 (6), 975-981. https://doi.org/10.1039/C2JA30109A.

(60) Coffer, M. T.; Shaw, C. F.; Hormann, A. L.; Mirabelli, C. K.; Crooke, S. T. Thiol Competition for Et3PAuS-Albumin: A Nonenzymatic Mechanism for Et3PO Formation. Journal Of Inorganic Biochemistry 1987, 30 (3), 177-187. https://doi.org/10.1016/0162-0134(87)80062-4.

(61) Bachman, R. E.; Bodolosky-Bettis, S. A.; Pyle, C. J.; Gray, M. A. Reversible Oxidative Addition and Reductive Elimination of Fluorinated Disulfides at Gold(I) Thiolate Complexes: A New Ligand Exchange Mechanism. JACS 2008, 130 (43), 14303-14310. https://doi.org/10.1021/ja805266r.
(62) Reglinski, J.; Hoey, S.; Smith, W. E. Exchange Reactions between Disulphides and Myocrisin: An in Vitro Model for a Mechanism in Chrysotherapy. Inorg Chim Acta 1988, 152 (4), 261-264. https://doi.org/10.1016/S0020-1693(00)91479-8.

(63) Thomas, R. C.; Reed, L.J. Disulfide Polymers of DL- $\alpha$-Lipoic Acid. JACS 1956, 78 (23), 6148-6149. https://doi.org/10.1021/ja01604a053. 